\title{
Some Problems of Modeling the Volume Laser- induced Phase Transformations in Relaxed Optics
}

\author{
Petro Trokhimchuck \\ Dept. Theor. @ Math. Physics, Lesya Ukrayinka \\ Eastern European National University, \\ Lutsk, Ukraine, 43025 \\ e-mail: trope1650@gmail.com
}

\begin{abstract}
The problems of creation the volume laser-induced phase transformation are discussed. Influences of processes the multiphoton absorption and impact ionization are observed. The proper models (Lugovoy-Prokhorov theory of moving focuses and modified Rayleygh model) are used for the explanation of receiving experimental data. Possible application the cascade model for the representation these results is analyzed too.
\end{abstract}

Index Terms - Relaxed Optics; impact ionization; styling; Lugovoy-Prokhorov theor;, Rayleygh model; cascade model

\section{INTRODUCTION}

Relaxed Optics (RO) is the chapter of modern physics of irreversible interaction light and matter $[1,2]$. Necessity of creation RO is caused of technological applications of laser radiation (laser annealing, laser implantation and other [2]). Phenomenological energy-time and electromagnetic classifications of processes and phenomena of interaction light and matter are basis of RO. According to energy-time classification we have three types of processes and phenomena: kinetic (mainly quantum first-order processes); dynamic (mainly wave second-order processes) and mixing kineticdynamic or dynamic-kinetic processes. Properly to this concept we can have three types phase transformations: kinetic, dynamic and more complex cascade kinetic-dynamic or dynamic-kinetic processes. Really it is photochemical, plasmic, thermal and mixing phenomena [2].

Thermal and plasmic processes are the field processes and for the light scattering in matter it are second order processes. Therefore the time of formation of these processes is more as time of first-order quantum processes (photochemical or photocrystall chemical). Hierarchy of this times is next: time of optical excitation $-10^{-18}-10^{-15} \mathrm{~s}$, time of local (quantum) electromagnetic relaxation $-10^{-15}-10^{-12} \mathrm{~s}$, time of generation of plasmic oscillations $-10^{-13}-10^{-10} \mathrm{~s}$, "thermal" times of heating and cooling $-10^{-9}-10^{-5} \mathrm{~s}$ [2]. First two processes are primary and quantum processes, last two processes are secondary.

Primary processes are caused of mechanisms of light scattering and local microscopic relaxation, secondary macroscopic relaxation mechanisms. Therefore we must include these results for the explanation of real picture of interaction light and matter.
The spectra of possible methods of modeling the phase transformations in Relaxed Optics are richer as theory of phase transitions. For this modeling we can include the physicalchemical kinetic methods and can represent phase transformations as structural "chemical" reactions. This method may be physical, chemical and physical-chemical [2]

\section{II.BASIC RESULTS AND DISCUSSIONS}

First system research of volume laser-induced phase transformations (breakdown and mechanical damage) in dielectrics (glasses) was realized by B. Sharma [3]. He selected basic peculiarities of interaction the laser irradiation with volume of solid.

According to [3] basic possible mechanisms for energy conversion are:

1. Electrostriction, which is included strict light preassure and electrostrictive preassure.

2. Stimulated Brillouin scattering.

In this Ph. D. Thesis [3] next important questions were analyzed:

1. Multiphoton ionization, including tunnelling theory and perturbation theory.

2. Classical microwave breakdown theory.

3. Absorption by inverse bremstrahlung.

4. The effect initiating of initiating electrons and electron multiplication.

5. Absorption of irradiation in focal volume.

B. Sharma research the laser-induced breakdown in glasees, fused quartz and crystalline quartz [3]. He was possibility to measure the change of phoconductivity and other optical properties of irradiated matter. But these researches shown some basic peculiarities of irreversible volume processes the interaction light and matter.

Other experimental data may be represented as "irreversible traces" of Nonlinear Optical processes [4 - 8].

Basic peculiarities of the creation the volume laser-induced phase transformations are represented in Fig. 1 - Fig. 32 [9, $10]$.

In $[9,10]$ for minituarization of receiving structures of crystals $4 \mathrm{H}-\mathrm{SiC}$ were irradiated by pulses of femtosecond laser (duration of pulses $130 \mathrm{fs}$, wavelength $800 \mathrm{~nm}$, frequency of pulses $1 \mathrm{kHz}$, density of energy 200-300 $\mathrm{nJ} /$ pulse) with help microscope. 
Conditions of irradiation are represented in Fig. 1 ((a), (b)) [9]. Femtosecond laser pulses were irradiated along the lines inside $4 \mathrm{H}-\mathrm{SiC}$ single crystals at a depth of $30 \mu \mathrm{m}$ by moving the sample at a scan speed of $10 \mu \mathrm{m} / \mathrm{s}$. The laser beam was irradiated at a right angle to the (0001) surface of the crystal.

The irradiated lines were almost parallel to the $[1 \overline{1} 00]$ direction. A schematic illustration of the laser-irradiated pattern is shown in Fig. 1 (a). The distance between neighboring lines was $20 \mu \mathrm{m}$.

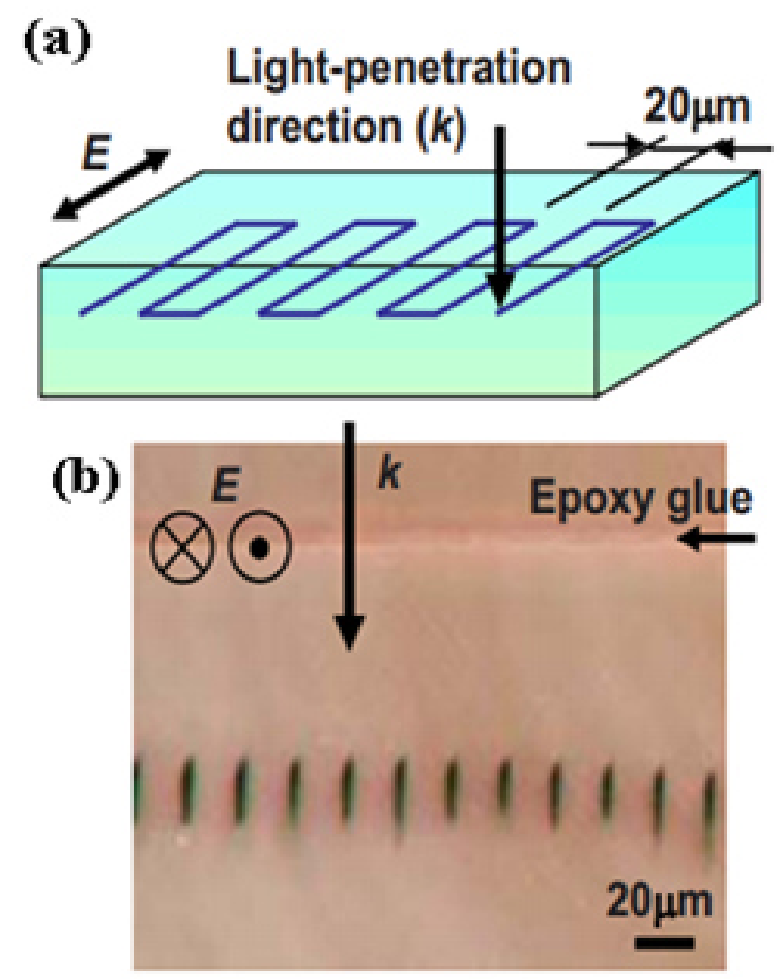

Fig. 1. Geometry of irradiation of $4 \mathrm{H}-\mathrm{SiC}[9,10]$. (a) Schematic illustration of the laser irradiated pattern. The light propagation direction $(\mathrm{k})$ and electric field (E) are shown. (b) Optical micrograph of the mechanically thinned sample to show cross sections of laser-irradiated lines $(200 \mathrm{~nJ} /$ pulse $)$.

Geometry of this experiment allows selecting the creation of elementary act of interaction light and matter.

Bright-field TEM (transmission electron microscopy) image of the cross section of a line written with a pulse energy of $300 \mathrm{~nJ} /$ pulse is shown on Fig. 2 and Fig. $3[9,10]$.

The geometry of this experiment is corresponded to optimal regime of modeling Lugovoy-Prokhorov scenario of observation the moving focuses [11, 12] (Fig. 2a). First stage is the result of microscope focusing and next four stages may be represented as "irreversible trace" of moving focuses [11, $12]$.

In addition the angle from peak of Fig. 2 (c) is corresponded to Cherenkov angle too and therefore this result may be representing as irreversible trace of Cherenkov picture in matter [13-15].

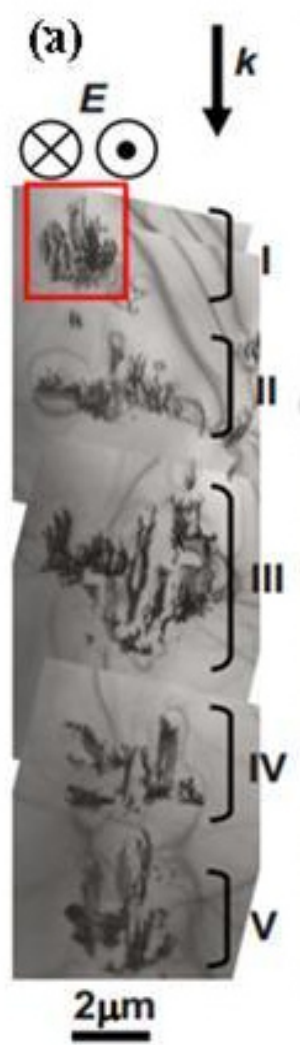

(b)

(c)
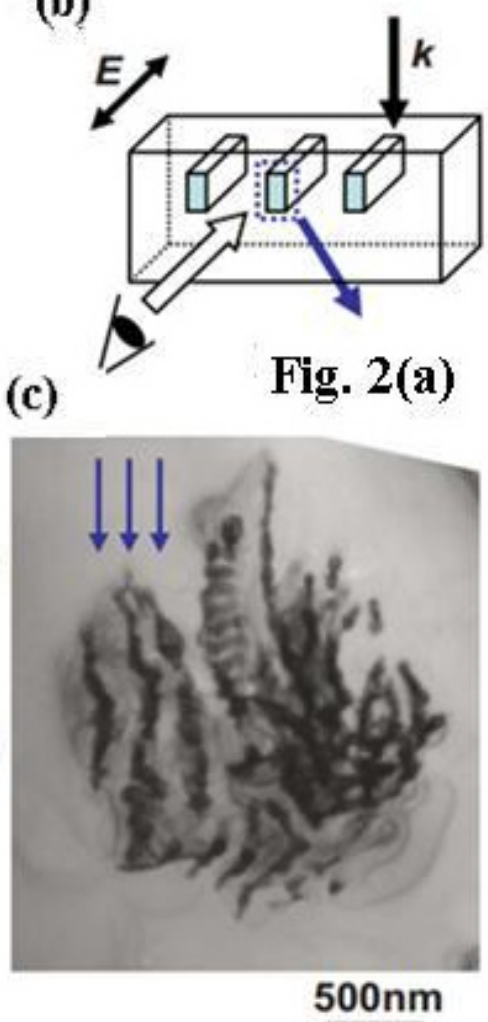

Fig. 2. Laser-induced damages cascades in $4 \mathrm{H}-\mathrm{SiC}[9,10]$. (a) Brightfield TEM image of the cross section of a line written with pulse energy of $300 \mathrm{~nJ} /$ pulse. (b) Schematic illustration of a geometric relationship between the irradiated line and the cross-sectional micrograph. (e) Magnified image of a rectangular area in (a).

In contrast to the formation of surface periodical structures three-dimensional periodic structures were obtained in this case. Sectional area of these structures was $\sim 22 \mu \mathrm{m}$, the depth of $\sim 50 \mu \mathrm{m}$. As seen from Fig. 2(a) we have five stages disordered regions, which are located at a distance from 2 to 4 $\mu m$ apart vertically $[9,10]$. Branches themselves in this case have a thickness from 150 to $300 \mathrm{~nm}$. In this case there are lines in the irradiated nanocavity spherical diameter of from $10 \mathrm{~nm}$ to $20 \mathrm{~nm}$. In this case irradiated structures have crystallographic symmetry of the initial structure.

Irregular motion of charges in the polarized atom (molecule) is a microscopic brake mechanism, which is corresponded to Cherenkov radiation. Thus, this explanation of the mechanism of Cherenkov radiation allows telling us, what in this phenomenon, similarly as well as in all processes of radiation the charge in electric or magnetic fields, the basic sources of radiation are accelerated moving matter [8, $13-15]$.

A presence of large amounts of atoms, which slow down a propagation of electromagnetic is necessary condition of existence of this mechanism. Wavelengths of these waves must be considerably more as interatomic distance in matter. For separate atom or a few atoms, being in a vacuum, such process 
is impossible, as their polarization will change with velocity of light in a vacuum [8].

More detail information about processes, which are generated in first two stages, represents in Fig. 3 ((a) - (c)) [9, $10]$.

(a)

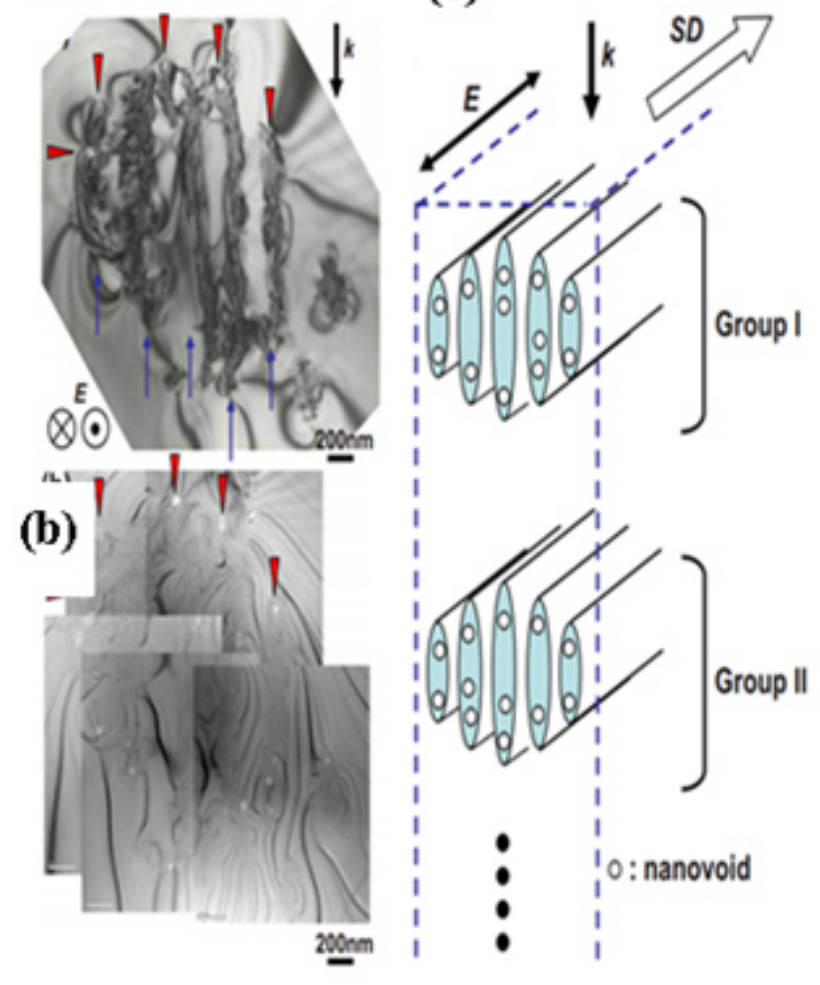

Fig. 3. Laser-modified layers with a spacing of $150 \mathrm{~nm}$ are indicated by arrows. (f) Bright-field TEM image of a portion of the cross section of a line written with a pulse energy of $200 \mathrm{~nJ} /$ pulse. (g) Zero-loss image of a same area as in (f) with nanovoids appearing as bright areas. Correspondence with (f) is found by noting the arrowheads in both micrographs. (h) Schematic illustrations of the microstructure of a laser modified line. Light-propagation direction (k), electric field (E), and scan direction (SD) are shown. Only two groups (groups I and II) of the laser-modified microstructure are drawn [9, $10]$.

Other picture we have for laser-induced volume phase transformations in SiC (Fig. 1 - Fig. 3). Explanation of the experimental data, which are shown in Fig. 2 and Fig. 3 is based on nanoplasmic model $[9,10]$. The emergence nanovoids explained on the basis of the explosive mechanism. However, the same result can be explained by the formation of vacancy clusters, especially those sizes of nanovoids same are equivalence to sizes of nanoclusters. Nanovoids, as a rule, are formed between the most modified regions, i.e. in these areas there are sinks of vacancies [32], which form the nanovoids or vacancies clasters.

Nanovoids may be represented as results of the laserinduced laser-induce break-down and creation of cavitation bubbles [16 - 19] too. The light pressure may be determined with help of next formula [3]

$$
p_{0}=\frac{E_{i r}}{\tau_{i} c S},
$$

where $E$ - energy of irradiation, $\tau_{i}$ - pulse duration, $S$ - area of irradiation zone, $c$ - speed of light. For circle symmetry [1]

$$
S=\pi r^{2}
$$

where $r$-radius of laser spot.

For the estimations of maximal radius of nanovoids we must use modified Rayleygh formula [1, 17]

$$
R_{\max } \simeq \frac{T_{c}}{0,915} \sqrt{\frac{p_{0}}{\rho_{0}}},
$$

where $T_{c}$ - the time of creation the nanovoid (bubble), $\rho_{0}-$ the density of irradiated matter.

Time $T_{c}$ may be determined as [1]

$$
T_{c} \simeq \frac{d_{c}}{\vartheta_{s}}
$$

where $d_{c}$ - characterized size of nanovoid (cavitation bubble), $\vartheta_{s}-$ speed of sound. For the spherical symmetry $d_{c}=2 R$, where $\mathrm{R}$ is radius of nanovoid.

The speed of sound may be determined as $[1,20,21]$

$$
\vartheta_{s}=\sqrt{\frac{E}{\rho_{o}}},
$$

where E - Young module [20, 21].

The finished formula for determination $R_{\max }$ has next form

$$
R_{\max } \simeq \frac{2 R}{0,915 r} \sqrt{\frac{E_{i r}}{\pi \tau_{i} c E}} .
$$

If we substitute $r=250 \mathrm{~nm}, R=10 \mathrm{~nm}, E=600 \mathrm{GPa}$ [20, 21], $E_{i r}=130 \mathrm{~nJ}, \tau_{i}=130 \mathrm{ps}, \mathrm{c}=3 \cdot 10^{8} \mathrm{~m} / \mathrm{s}$, than have $R_{\max }=11$ $n m$.

The speed of shock waves for femtosecond regime od irradiation is less as speed of sound. But we have two speeds of sound in elastic body: longitudinal $\vartheta_{l s}$ and transversal $\vartheta_{t s}$ $[20,21]$. Its values are determined with next formulas

$$
\vartheta_{l s}=\sqrt{\frac{E(1-v)}{\rho_{o}(1+v)(1-2 v)}},
$$

and

$$
\vartheta_{t s}=\sqrt{\frac{E}{2 \rho_{o}(1+v)}},
$$

where $v$ - Poisson's ratio $[20,21]$. The ratio between of these two speeds is equaled 


$$
\alpha=\frac{\vartheta_{t s}}{\vartheta_{l s}}=\sqrt{\frac{(1-2 v)}{2(1-v)}} .
$$

But this ratio must be true for shock waves too. Therefore for silicon carbide for $v=0,45[20,21] \alpha=0,33$. Roughly speaking last ratio is determined the step of ellipsoidal forms of our nanovoids (Fig. 3). This macroscopic model allows explaining basic peculiarities of experimental data of Fig. 3.

The creation of five groups of nanovoids (Fig. 2a) is caused of "moving" focus in irradiated matter, which is connecting with processes of blooming at the irradiation time $[7,8]$.

Formula (6) may be represented in more précised form. Expression for $T_{\mathrm{c}}$ (formula (4) must have next form

$$
T_{c} \simeq \frac{d_{c}}{\vartheta_{l s}-\vartheta_{t s}} .
$$

After substitution relations (6a) and (6b) we have

$$
T_{c} \simeq \sqrt{\frac{2 \rho_{o}(1+v)(1-2 v) d_{c}}{E}} .
$$
have

After substitution formulas (10 band (4c) to relation (3) we

$$
R_{\max 1} \simeq R_{\max } \sqrt{(1+v)(1-2 v)} \simeq 0,12 R_{\max }
$$

As we see the formula (6) explains proper experimental data more precisely as formula (6c). It is result of fact that we don't include the change of Young module and Poisson ratio in the process of irradiation. Second cause is next: we must include in (6) and (6a) the speed of shock wave, which is more as speed of sound.

Thus for the explanation laser-induced volume phase transformations we must use models of laser-induced breakdown, moving focuses, radiation physics of solid state and shock waves.

For more detail modeling we must include the microscopic mechanisms of creation these phase transformations.

\section{CONCLUSIONS}

1. Basic peculiarities of volume laser-induced transformations in matter are analyzed.

2. Experimental data about iunteraction of series the femtosecond laser irradiation and $4 \mathrm{H}-\mathrm{SiC}$ are observed.

3. Possiible influence of microscopic mechanism of Cherenkov radiation on processes of volume lasere-induced phase transformations in matter is discussed.

4. Was shown, that Lugovoy-Prokhorov theory of moving focuses may be used for the explanation of the creation the volume laser-induced periodical structures.

5. Modified Rayleygh model is created and used for explanation the sizes of laser-induced nanovoids.

\section{REFERENCES}

[1] P. Trokhimchuck, "Problems of modeling the phase transformations in Nonlinear and Relaxed Optics,” Int J. Eng. Res. @ Developm. (IJERD), vol.143, is.2, 2018, pp. 48-61.

[2] P. Trokhimchuck, "Relaxed Optics: Realities and Perspectives," Saarbrukken: Lambert Academic Publishing, 2016, 260 p.

[3] B. S. Sharma, "Laser-induced dielectric breakdown and mechanical damage in silicate glasses," Burnaby: Ph. D. Thesis Simon Fraser University, 1968, $123 \mathrm{p}$.

[4] R. W. Boyd, "Nonlinear optics," Amsterdam a.o.: Academic Press, $2003,578 \mathrm{p}$.

[5] Y. R. Shen , "The Principles of Nonlinear Optics," New York: John Wiley \& Sons, , 1984, 576 p.

[6] N. I. Koroteev and I. L. Shumay, "Physics of power laser irradiation," Moscow:: Nauka, 1991., 312 p. (In Russian)

[7] P. P. Trokhimchuck, "Problems of reradiation and reabsorption in Nonlinear and Relaxed Optics," Int. J. Adv. Res. Phys. Sc. (IJARPS), vol.4(2), pp. 37-50, 2017.

[8] P. Trokhimchuck, "Nonlinear and Relaxed Optical Processes. Problems of Interactions," Lutsk: Vezha-Print, 2013, 280 p.

[9] T. Okada, T. Tomita, S. Matsuo , S. Hashimoto, R. Kashino and T. Ito, "Formation of nanovoids in femtosecond laser irradiated single crystal silicon carbide," Material Science Forum, vol. 725, pp. 19 - 22, 2012.

[10] T. Okada, T. Tomita, S. Matsuo , S. Hashimoto, Y. Ishida, S. Kiyama and T. Takahashi, "Formation of periodic strain layers associated with nanovoids inside a silicon carbide single crystal induced by femtosecond laser irradiation,” J. Appl. Phys., v. 106(5), pp.054307, 5 p., 2009.

[11] V. N. Lugovoi, A. M. Prokhorov, "Possible Explanation of the Smallscale Self- focusing Filaments," JETP Letters, vol. 7, Is. 5, 1967, pp. 117-119., (In Russian)

[12] "Self-Focusing: Past and Present," eds. R. W. Boyd, S. G. Lukishova, Y.-R. Shen, Springer Series: Topics in Applied Physics, Vol. 114., NY: Springer, 2009, $605 \mathrm{p}$.

[13] N. Bohr, "The penetration of atomic particles through matter," Moscow: Inostrannaya literatura, 1950, $152 \mathrm{p}$. (In Russian)

[14] I. M. Frank, "Vavilov-Cherenkov radiation. Theoretical aspects," Moscow: Nauka, 1988, 286 p. (In Russian)

[15] A. P. Kobzev, "Mechanism of Vavilov-Cherenkov radiation," Elementary particles and atomic nuclear, vol.41, No.3, 2010, pp.830-867 (In Russian)

[16] Byun Ki-Taek and Kwak Ho-Young, "A model of laser-induced cavitation," Japanese Journal of Applied Physics, vol. 43(2), pp. 6216302004

[17] T. Juhash, G. A. Kastis, C. Suares, Z. Borand and W. E. Bron, "TimeResolved Observations of Shock Waves and Cavitation Bubbles Generated by Femtosecond Laser Pulses in Corneal Tissue and Water," Lasers in Surgery and Medicine, vol. 19, pp. 23 - 31, 1996.

[18] W. Lauteborn and T. Kurz, "Physics of bubble oscillations," Rep. Progr. Phys., vol. 77. 106501, $88,2010$.

[19] F. V. Potemkin and E. I. Mareev, "Shock waves and cavitation bubbles dynamics as a function of the tightly focused femtosecond laser energy in distilled water and acetone," Scientific Notes of Physical Faculty. Mikhail Lomonosov Moscow State University, 133401, 9, 2013 (In Russian)

[20] S. M. Ryndya, "Peculiarities of Thin Films SiC structure, which is formatted on $\mathrm{Si}$ and $\mathrm{Al}_{2} \mathrm{O}_{3}$ substrates by method of pulse laser precipitation.," Moscow: Ph. D. Thesis, L. Ya Karpov Scintific Research Physica-Chemical Institute, 2014, 158 p. (In Russian)

[21] D. O. Moskovskikh, "Production of Submicrometer Powder of Silicon Carbide and Nanostructural Ceramics on its Basis," Moscow: Ph. D. Thesis National Research Technological University Steel and Alloys, 2015, 166 p. (In Russian) 